\title{
XL. Catalogue of corresponding eclipses at one period distance
}

\section{Mr. Thomas Yeates}

To cite this article: Mr. Thomas Yeates (1820) XL. Catalogue of corresponding eclipses at one period distance, Philosophical Magazine Series 1, 56:270, 278-282, DOI:

10.1080/14786442008652403

To link to this article: http://dx.doi.org/10.1080/14786442008652403

册 Published online: 23 Jul 2009.

Submit your article to this journal

Џ Article views: 2

Q View related articles $\square$ 


\section{$[278]$}

XL. Catalogue of corresponding Eclipses at one Period Distance. By Mr. Thomas Yeates.

[Continued from vol. 55 , p. 247.]

A.D. Where observed. D. H. M. A. D.

D. H.

$1 \odot$ Pekin June $10 \quad 1 \quad 10 \quad 913 \odot \odot$ June 7 9.

$\begin{array}{lllllllllll}5 & \odot & \text { Rome } & \text { March } 28 & 4 & 13 & 917 & \odot & \text { Sept. } 5 & 0 & M\end{array}$

14 D PannoniaSept. $2617 \quad 15 \quad 926$ D Sept. $24 \quad 9 \frac{1}{2}$ M.

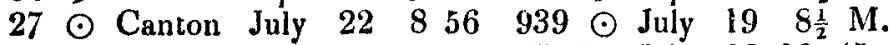

Paris July $18 \quad 1945$

$30 \odot$ Canton Nov. $131920 \quad 942 \odot$ Nov. $11 \quad 6 \frac{x}{2}$ M.

$40 \odot$ Pekin April $30 \quad 5 \quad 50 \quad 952 \odot$ April $2610 \frac{1}{2}$ N.

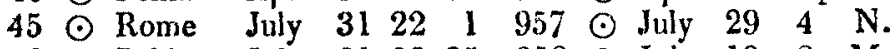

$46 \odot$ Pekin July $21 \quad 22 \quad 25 \quad 958$ ○ July $19 \quad 8 \quad M$.

D Rome Dec. $31 \quad 952 \quad D$ Dec. $2810 \frac{x}{2} \mathrm{~N}$.

$49 \odot$ Pekin May $20 \quad 7 \quad 16 \quad 961 \odot$ May $17 \quad 8 \frac{1}{2} \quad M$.

Rheims May $16 \quad 2013$

$53 \odot$ Canton March $82042 \quad 965 \odot$ March $6 \quad 3 \frac{1}{2} \mathrm{M}$.

$55 \odot$ Pekin July $12 \quad 21 \quad 50 \quad 967 \quad \odot$ July $10 \quad 6 \frac{x}{2} M$.

$56 \odot$ Canton Dec. $25 \quad 028968 \odot$ Dec. $22 \quad 9 \quad M$.

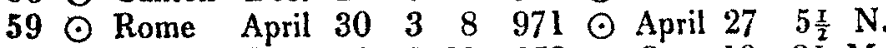

$60 \odot$ Canton Oct. $13 \quad 331 \quad 972 \odot$ Oct. $10 \quad 3 \frac{1}{2} \mathrm{M}$.

$65 \odot$ Canton Dec. 152150 , 977. $\odot$ Dec. $13 \quad 8 \frac{1}{2} \mathrm{M}$.

69 D Rome Oct, $181043 \quad 981$ D Oct. $16 \quad 3 \quad$ M.

$70 \odot$ Canton Sept. $22 \quad 21 \quad 13 \quad 982 \odot$ Sept. $20 \quad 3 \quad M$.

71 D) Rome March. 4832983 D March $111 \frac{\mathrm{r}}{2} \mathrm{~N}$.

$95 \odot$ Ephesus May 21 $1007 \odot$ May $19 \quad 8 \quad$ M.

125 D Alexand. April $5 \quad 9 \quad 161037$ D April $211 \frac{1}{2} \mathrm{~N}$.

Paris $\odot$ April 172045

133 D Alexand. May $61144 \quad 1045$ D May $3 \quad 9 \frac{1}{2}$ N.

134 Alexand. Oct. $2011 \quad 5 \quad 1046 \quad$ Oct. $17 \quad 7 \frac{1}{2} \mathrm{~N}$.

136 D Alexand. March 515561048 D March $3 \quad 6 \frac{1}{2} \mathrm{M}$.

$237 \odot$ Bologna April $12 \odot 1149 \odot$ April $12 \quad 9 \frac{\mathrm{I}}{2} \mathrm{~N}$.

$238 \odot$ Rome April $120201150 \odot$ March

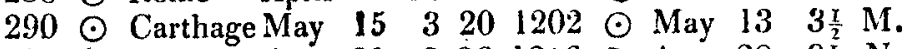

304 D Rome Aug. $31 \quad 9361216$ D Aug. $28 \quad 9 \frac{\mathrm{l}}{2} \mathrm{~N}$.

$316 \odot$ Constan. Dec. $3019531228 \odot$ Dec. $28 \quad 8$ M. Naples Dec. $27 \quad 955$

$334 \odot$ Toledo July 17 Noon $1246 \odot$ July $14 \quad 1 \quad N$. $348 \odot \odot$ Constan. Oct. $8 \begin{array}{lllllllll}19 & 24 & 1260 & \odot & \text { Oct. } & 6 & 12 & \text { N. }\end{array}$ $360 \odot$ Ispahan Aug. $2718 \quad 0 \quad 1272 \odot$ Aug. $25 \quad 1 \frac{1}{2}$ M. Vienna D Aug. $10 \quad 727$

364 Alexand. Nov, $251524 \quad 1276$ D Nov. $22 \quad 2 \frac{1}{2}$ M. Vienna Nov, 22150 
Catalogue of corresponding Eclipses at one Period Distance. 279

A.D. Where observed. D. H. M. A.D.

D. $\mathrm{H}$.

$401 \mathrm{D}$ Rome June 11 1313 D June 9 9 $\mathrm{M}$. D Rome Dec. 61215 D Dea $3 \quad 8 \frac{1}{2} \mathrm{~N}$. Torcello Dec. 3858

402 D Rome June I 8431314 D May $30 \quad 2 \frac{1}{2}$ M. $\odot$ Rome Nov. $102033 \quad \odot$ Nov. 822 N.

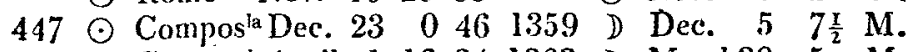
451 D Composia April 116341363 D March30 5 M. D Compos ${ }^{\mathrm{la} S e p t .26} 630 \quad$ D Sept. $23 \quad 8 \frac{\mathrm{x}}{2} \mathrm{~N}$. $458 \odot$ Chaves May $2723161370 \odot$ May $254 \frac{\mathrm{I}}{2} \mathrm{~N}$. 462 D Compos ${ }^{i 2}$ March I $1321374 \quad D$ Feb. $27 \quad 7 \frac{1}{2} \mathrm{M}$. $464 \odot$ Chaves July $1919 \quad 1 \quad 1376 \odot$ July $17 \quad 8 \frac{x}{2}$ M. $484 \odot$ Constan.Jan. $131953.1396 \odot$ Jan. $1111 \mathrm{M}$. Augsburg Jan. $11 \quad 0 \quad 16$

$456 \odot$ Constan. May $19 \quad 1 \quad 101398 \odot$ May $16 \quad 5 \quad N$. $497 \odot$ Constan. April $18 \quad 6 \quad 5 \quad 1409 \odot$ April $15 \quad 1 \frac{1}{2} \mathrm{~N}$. Constantinople April $15 \quad 31$

$512 \odot$ Constan. June $282381424 \odot$ June $26 \quad 2 \frac{1}{2} \mathrm{~N}$. Wittemburg June $26 \quad 357$

$538 \odot$ England Feb. $141901450 \odot$ Feb. $12 \quad 1 \quad \mathrm{~N}$. $540 \odot$ London June 1920 15 $1452 \odot$ June $17 \quad 3 \quad \mathrm{~N}$. $\begin{array}{lllllllll}577 & \text { D Tours Dec. } 10 & 17 & 28 & 1489 & \text { D Dec. } & 8 & 5 & \text { M. }\end{array}$ R. C.* Dec. 71741

581 D Paris April 413331493 D April 21 M. R.C. April 1140

582 D Paris Sept. 1712411494 D Sept. $15 \quad 6 \frac{1}{2} \mathrm{M}$. R.C. Sept. 141945

590 Daris Oct. 186301502 D Oct. $1511 \mathrm{~N}$. R.C. Oct. 151220

$592 \odot$ Constan. Mar. $182261504 \odot$ Marchl6 $1 \frac{1}{2} N$. $603 \odot$ Paris Aug. $12 \quad 3 \quad 31515 \odot$ Aug. $99^{2} \mathrm{~N}$. 622 D Constan. Feb. 111281534 D Jan. $30 \quad 1 \frac{1}{2} \mathrm{M}$. R.C. Jan. 291425

$644 \odot$ Paris Nov. $50301556 \odot$ Nov. $2 \quad 8 \quad \mathrm{M}$. New Style.

680 Paris June 1712301592 D June 241013 683 D Paris April 1611301595 D April $24 \quad 412$ $693 \odot$ Constan. Oct. $42354 \quad 1605 \odot$ Oct. $12 \quad 232$ 716 Constan. Jan. 13701628 D Jan. 201011 $718 \odot$ Constan. June $3 \quad 1 \quad 151630 \odot$ June $10 \quad 7 \quad 47$ $733 \odot$ England Aug. 1320 0 $1645 \odot$ Aug. $21 \quad 035$ 734 D England Jan. $2314 \quad 01646$ D Jan. 301811

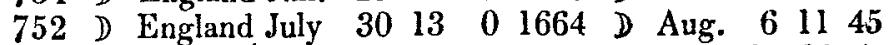
$753 \odot$ England June $\quad S 22 \quad 0 \quad 1665 \quad D$ July 2611 A. $\begin{array}{llllll}D & \text { England Jan. } 2313 & 0 & \text { D Jan. } 301847\end{array}$

* Ricciolus's Catalogue, 
280 Catalogue of corresponding Eclipses at one Period Distance.

A.D. Where observed.

D. H. M. A.D.

N. S. D. H.

$760 \odot$ Englatid Ang. $15 \quad 4 \quad 0 \quad 1672$

○ Aug. $22 \quad 643$

D London Aug. $30 \quad 550$

Sept. 61854

$764 \odot$ England June 4 noon. $1676 \odot$ June 102126

$770 \mathrm{D}$ London Feb. 147.121682 D Feb. $21 \quad 11 \mathrm{~N}$.

774 D Rome Nov. $221437 \quad 1686$ D Nov. $2912 \quad 22$

784 D London Nov. 11421696 D Nov. $912 \quad 22$

$787 \odot$ Constan. Sept. $1420431699 \odot$ Sept. 232238

796 D Constan. March27 16221708 D April 5 6

800 D Rome Jan. $15 \quad 9 \quad 001712$ D Jan. $23 \quad 8 \quad$ A.

$807 \odot$ Angoul $^{\text {me }}$ Feb. $102124 \quad 1719 \odot$ Feb. $19 \quad 7 \quad M$.

D) Paris Feb. 25 l3 43 D March $6 \quad 8$ M.

D Paris Aug. 21 $1020 \quad$ D Aug. $29 \quad 9 \quad$ A.

$809 \odot$ Paris July $1521331721 \quad \odot$ July $24 \quad 9 \quad$ M.

D Paris Dec. 25 S $0 \quad$ $\odot$ Dec. 19 l $M$.

810 D Paris June $20 \quad 8 \quad 01722 \quad D$ June $29 \quad 3 \quad M$.

$\odot$ Paris Nov. $30 \quad 0 \quad 12 \quad \odot$ Dec. $3 \quad 3 \quad A$.

D Paris Dec. $14 \quad 8 \quad 0 \quad$ D Dec. 2244 A.

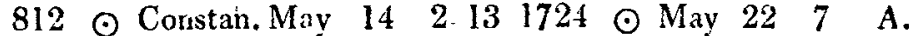

$813 \odot$ Cappad May $^{a} \quad 17 \quad 15 \quad 1725$ ○ May $1210 \frac{\mathrm{r}}{2} \mathrm{M}$.

817 D Paris Feb. 5 5 $42 \quad 1729$ D Feb. $13 \quad 6 \quad$ A.

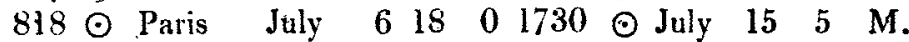

820 D Paris Nov. $23 \quad 6 \quad 26 \quad 1732$ D Dec. $110 \quad$ N.

824 D Paris March18 $755 \quad 1736$ D March26 $12 \quad$ N.

828 D Paris Dec. 2413451740 D

831 D Paris Aprił $30 \quad 6$ i9 1743 D May 8 3 $\frac{1}{2}$ A.

$\odot$ Paris $\quad$ May $1523 \quad 0 \quad 0$ May $23 \quad 6 \quad$ A.

D Paris Oct. $241118 \quad$ D Nov. 233 M.

832 D Paris April $18 \quad 9 \quad 0 \quad 1744 \quad$ D April $26 \quad 9 \quad$ A.

$840 \odot$ Paris May $423221752 \odot$ May $13 \quad 8 \quad \mathrm{~A}$.

$841 \odot$ Paris $\quad$ Oct. $1718581753 \quad \odot$ Oct. $2610 \mathrm{M}$.

842 D) Paris March29 14381754 D April 7 8 $\frac{1}{2} \mathrm{M}$.

843 D Paris March19 $7 \quad 1 \quad 1755$ D March28 1 M.

861 D Paris March29 $15 \quad 7 \quad 1773$ D April $7 \quad 9 \quad M$.

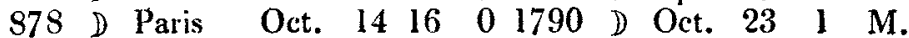

883 D Arracta July $23 \quad 7 \quad 14 \quad 1795$ D

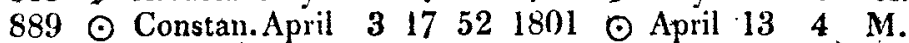

$891 \odot$ Constan. Aug. 723481803 ○ Aug. $17 \quad 8 \quad M$.

901 D. Arracta Aug. $215 \quad 7 \quad 1813$ D Aug. $12 \quad 3 \quad M$.

904 London May 3111471816 D June $10 \quad 1 \quad M$.

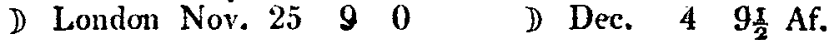

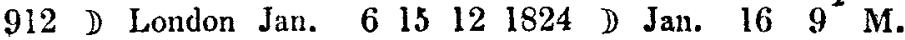

Note. All the historical eclipses in the above Catalogue are taken from Mr. Ferguson's Tabres, and their corresponding ones from L'Art de vérifier les Dates. Paris 1;85.

Series 


\section{[ 281 ]}

Series of corresponding Eelipses in a Lunar Cycle at one Period Distance.

[Appendix to page 445, vol. 55.]

A.D. O. Style. D. H. M. A.D. N. Style. D. H. M.

889 March21 $230 \mathrm{M}$. ○ April $4430 \mathrm{M}$.

$\odot \overline{\text { Sept. }} 1 \overline{3} \overline{4}-\overline{30} \mathrm{M}$.

890 Marchi0 Noon.

$\odot$ Aug. $1910 \quad 0 \mathrm{M}$.

- Sept. 250 Af.

$891 \odot$ Feb. 1240 Af.

$\odot$ Aug. $81030 \mathrm{M}$.

892

D Aug. $23930 \mathrm{M}$.

O Feb. $-\overline{2}$ 8 0 M.

D July $13 \quad 3 \quad 30$ Af.

893 J Jan. $6530 \mathrm{M}$.

$\odot$ June 1750 Af.

- July 21045 Af.

$894 \underset{\odot \text { June }}{7} \frac{1}{10} \frac{-}{0} \mathrm{M}$.

D June $22 \quad 0 \quad 30$

$\odot--16-$

Dec. $161130 \mathrm{M}$.

895

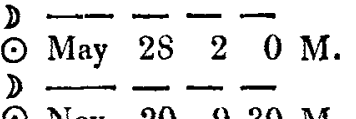

$\odot$ Nov. $20930 \mathrm{M}$.

$396 \mathrm{D}$ May 1. $230 \mathrm{M}$.

D Oct. $25 \quad 030 \mathrm{M}$.

$\odot$

April $5 \overline{11} 0$ Af

- Oct. $141145 \mathrm{M}$.

$898 \odot$ Mar. 26 I 0 M. D April $10110 \mathrm{M}$.

$D$ Oct. 3.30 Af.

$899 \bigcirc$ Mar. 151030 M.

D Aug. 2450 Af.

900 Feb. $18930 \mathrm{M}$.

- Aug. $13930 \mathrm{M}$.

$901 \odot$ Jan. $23630 \mathrm{M}$.

- Feb. $6100 \mathrm{M}$.

Vol. 56. No. 270. Oct. $18 \% 0$.
1801 Mar. $30530 \mathrm{M}$

○ April $13430 \mathrm{M}$.

$\odot$ Sept. $860 \mathrm{M}$.

- Sept. $22730 \mathrm{M}$.

1802 D March19 11 $30 \mathrm{M}$.

$\odot$ Aug. $25730 \mathrm{M}$.

Dept. $11110 \mathrm{~N}$.

$1503 \odot$ Feb. 21

$\odot$ Aug. $17830 \mathrm{M}$.

1804 D Jan. 26 $9 \overrightarrow{30}$ Af.

○ Feb. $111130 . \mathrm{M}$.

D July 22530 Af.

1805 Jan. $1590 \mathrm{M}$.

$\odot$ June 26110 Af.

- July 1190 Af.

- Jan. 4

1805 D Jan. $5000 \mathrm{M}$.

$\odot$ June $16 \quad 4 \quad 0$ Af.

D June $30 \quad 10 \quad 0$ Af.

$\odot$ Dec. $10230 \mathrm{M}$.

1807 D May $\overline{21} \overline{5} \overline{30}$ Af.

$\odot$ June $6530 \mathrm{M}$.

D Nov. $15830 \mathrm{M}$.

$\odot$ Nov. 29 Noon.

$1808-$ May $1080 \mathrm{M}$.

- Nov. 3990 M.

$\odot$ Nov. $1830 \mathrm{M}$.

$1809 \odot$ April 14

D April $3010 \mathrm{M}$.

Oct. $23 \quad 930$ M.

$1810 \stackrel{\bigcirc}{\odot}$ April $420 \mathrm{M}$.

$1811 \stackrel{\odot}{\mathrm{March} 24}-\overline{10}-\overline{0} \mathrm{M}$.

D Sept. 2110 Af.

1812 Feb. $2760 \mathrm{M}$.

- Aug. 2230 Af

$1813 \odot \mathrm{Feb} . \quad 1 \quad 9 \quad 0 \cdot \mathrm{M}$.

D Feb. $1590 \mathrm{M}$.

$\mathrm{N} n$

901 


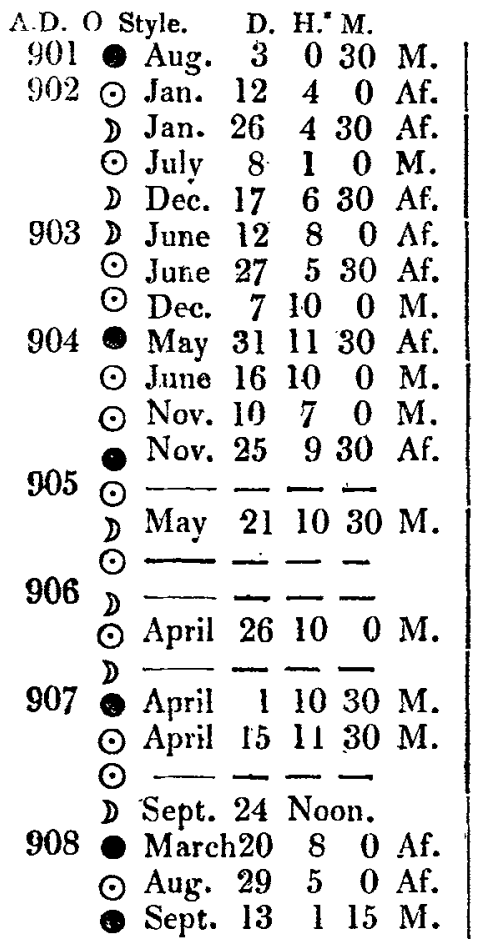

A.D. N. Style, D. H. M.

D Aug. $12 \quad 315 \mathrm{M}$.

$1814 \odot$ Jan. $21 \quad 230$ Af.

$\stackrel{\odot \text { July }}{17} \overline{7} \overline{0} \mathrm{M}$.

1915 Dec. $26 \quad 1130$ Af.

$\odot$ July $7000 \mathrm{M}$.

- Dec. 16 1 15 Af.

1816 June $10180 \mathrm{M}$.

$\odot \cdots---$

$\odot$ Nov. $191030 \mathrm{M}$.

- Dec. 490 Af.

$1817 \odot$ May $16 \quad 7 \quad 0 \quad M$.

D May $30 \quad 3 \quad 30$ Af.

$\odot$ Nov. $92280 \mathrm{M}$.

1818 D April $21 \quad 030 \mathrm{M}$. $\odot$ May $5730 \mathrm{M}$. D Oct. $14 \quad 6 \quad 0$ M.

1819 April 10 130 Af.

$\odot$ April 24 Noon.

$\odot$ Sept. 1910 Af. D Oct. $3 \quad 3 \quad 30$ Af. 1820 D Mar. $29.7 \quad 0$ Af. ( S Sept. 720 Af. D Sept.22 700 M.

Note. All the eclipses in this list are computed by the author of L'Art de vérifier les Dates. Paris 1785.

\section{On Lithography*.}

MR. HULLMANDELL ON LITHOGRAPHY.

$T_{\text {H B drawings I have the honour to present to the Society for }}$ promoting the Arts, Manufactures, and Commerce, are the fruits of an art invented some years ago in Germany, and but lately introduced into this country, at least in its present state of perfection. Its great advantage is that of enabling the artist to

* From the Transactions of the Saciety for the Encouragement of Arts, Masufactures, and Commerce, vol. xxxvii. The silver medal of the Society was voted to the author of the first of the subjoined papers, Mr. Hullmandell, of Great Marlborough-street, London, for the communication, and the specimens remain in the Society's Repository. The silver Isis medal was voted to Mr. Redman, of Maiden-lane, for another communication on the same sulject, which we also subjoin. 